\title{
EFFECTS OF SUPPLEMENTAL VISCOUS DAMPING ON SEISMIC RESPONSE OF ASYMMETRIC-PLAN SYSTEMS
}

\author{
RAKESH K. GOEL* \\ Department of Civil and Environmental Engineering, Cal Poly State University, San Luis Obispo, CA 93407, U.S.A.
}

\begin{abstract}
SUMMARY
Coupling between lateral and torsional motions may lead to much larger edge deformations in asymmetric-plan systems compared to systems with a symmetric plan. Supplemental viscous damping has been found to be effective in reducing deformations in the symmetric-plan system. This investigation examined how supplemental damping affects the edge deformations in asymmetric-plan systems. First, the parameters that characterize supplemental viscous damping and its plan-wise distribution were identified, and then the effects of these parameters on edge deformations were investigated. It was found that supplemental damping reduces edge deformations and that reductions by a factor of up three are feasible with proper selection of system parameters. Furthermore, viscous damping may be used to reduce edge deformations in asymmetric-plan systems to levels equal to or smaller than those in the corresponding symmetric-plan system. (C) 1998 John Wiley \& Sons, Ltd.
\end{abstract}

Earthquake Engng. Struct. Dyn., 27, 125-141 (1998)

KEY WORDS: asymmetric buildings; earthquake response; passive control; plan asymmetry; protective systems; seismic response; supplemental damping; torsion

\section{INTRODUCTION}

It is well known that asymmetric-plan buildings are especially vulnerable to earthquakes. It is therefore not surprising that numerous investigations in the past have focused on the earthquake behaviour of asymmetric-plan systems; references are available in reports by Hejal and Chopra, ${ }^{1}$ Goel and Chopra ${ }^{2}$ or in a state-of-the-art review paper by Rutenberg. ${ }^{3}$ As a result, there has been much improvement in our understanding of how plan asymmetry influences the response of buildings to earthquakes. Procedures to account for undesirable effects of plan asymmetry, such as increased force and ductility demands on lateral load-resisting elements, have been developed and incorporated into many seismic codes. ${ }^{4}$ However, additional research is needed to develop techniques that will control excessive earthquake-induced deformations in asymmetric-plan buildings. The excessive deformations may lead to premature failure in brittle, nonductile elements and may result in a sudden loss of the building's strength and stiffness leading to eventual failure. Excessive edge deformation may also cause pounding between closely spaced adjacent buildings.

In general, excessive deformation in asymmetric-plan buildings may be reduced by redistributing the stiffness and/or mass properties to minimize the stiffness eccentricity. For many new structures, such redistribution may be possible at an early design stage, but such redistribution may not always be feasible because of architectural or functional constraints. It may not be feasible for existing structures because of the significant inconvenience to the occupants.

\footnotetext{
* Correspondence to: R. K. Goel, Department of Civil and Environmental Engineering, California Polytechnic State University, San
} Luis Obispo, CA 93407, U.S.A. 
In recent years, the role of supplemental damping or energy dissipation devices in reducing the earthquake response of structures has been the subject of many investigations, ${ }^{5-18}$ a comprehensive summary is available elsewhere. ${ }^{8}$ Although these investigation have led to much improvement in our understanding of how such devices improve the sesimic performance of structures, they were primarily focused on the seismic behaviour of symmetric-plan systems. A few recent investigations have been concerned with the seismic behaviour of asymmetric-plan systems with supplemental devices. ${ }^{19-22}$ However, there has been a lack of effort towards developing a fundamental understanding of how these devices and their plan-wise distribution influence the lateral-torsional coupling in asymmetric-plan systems.

With this aim, the objectives of this exploratory investigation are (1) to identify the system parameters that control the seismic response of asymmetric-plan systems with fluid viscous dampers; and (2) to investigate the effects of the controlling parameters on edge deformations in asymmetric-plan systems. System behaviour is limited to the linearly elastic range. This simplifying assumption is suitable for investigating the seismic behaviour of asymmetric-plan systems with brittle, non-ductile resisting elements, that should remain nearly elastic during earthquake loading. The seismic response of asymmetric structures responding in the inelastic range will be investigated during a later phase of this research program.

\section{SYSTEM AND GROUND MOTION}

\section{One-storey system}

The system considered was the idealized one-storey building of Figure 1 consisting of a rigid deck supported by structural elements (wall, columns, moment-frames, braced-frames, etc.) in each of the two orthogonal directions. In addition, the system included fluid viscous dampers (FVDs) incorporated into the bracing system. The mass properties of the system were assumed to be symmetric about both the $X$ - and $Y$-axis whereas the stiffness and the damper properties were considered to be symmetric only about the $X$-axis.

The centre of mass $(\mathrm{CM})$ of the system was defined as the centroid of inertia forces when the system is subjected to a uniform translational acceleration in the direction under consideration. Since the mass was uniformly distributed about both the $X$-and $Y$-axis, the CM coincided with the geometric centre of the deck.

The centre of supplemental damping (CSD) was defined as the centroid of damper forces when the system is subjected to a uniform translational velocity in the direction under consideration. The lack of symmetry in the damper properties about the $Y$-axis was characterized by the supplemental damping eccentricity, $e_{\mathrm{sd}}$, defined as the distance between the CM and the CSD.

The centre of rigidity (CR) was defined as the point on the deck through which application of a static horizontal force causes no rotation of the deck. ${ }^{1}$ For the one-storey system considered in this investigation, $\mathrm{CR}$ was also the centroid of resisting forces in structural elements when the system is subjected to a uniform translational displacement in the direction under consideration. The lack of symmetry in the stiffness properties about the $Y$-axis was characterized by the stiffness eccentricities, $e$, defined as the distance between the $\mathrm{CM}$ and the CR. With both CM and CR defined, the edge that is on the same side of the CM as the CR was denoted as the stiff edge and the other edge was designated as the flexible edge (Figure 1(a)).

The corresponding symmetric-plan system was defined as a system with no FVDs and coincidental CM and CR but with relative locations and stiffnesses of all resisting elements identical to those in the asymmetric-plan system.

\section{Ground motion}

The ground motion considered is the North-South $\left(360^{\circ}\right)$ component recorded at the Sylmar County Hospital parking lot during the 1994 Northridge earthquake. The peak values of the ground acceleration, velocity and displacement recorded at this site were $826.8 \mathrm{~cm} / \mathrm{s}^{2}, 128.9 \mathrm{~cm} / \mathrm{s}$, and $32.55 \mathrm{~cm}$, respectively. 


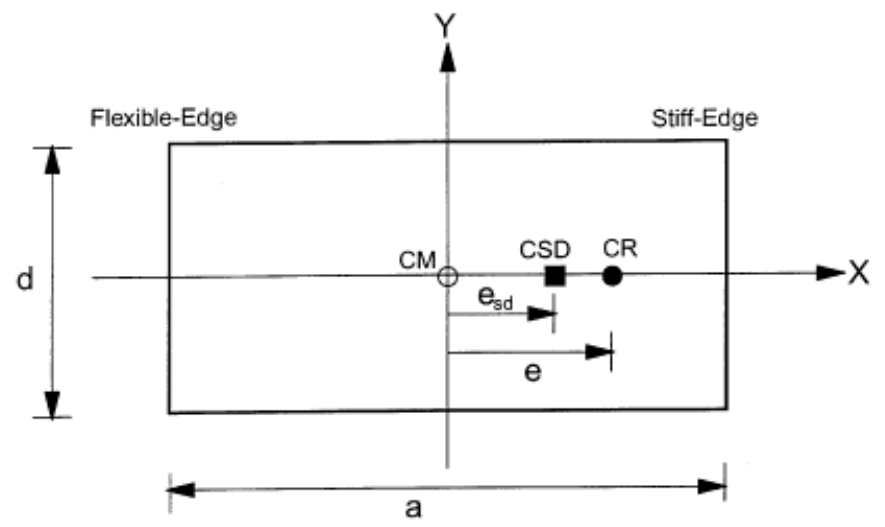

(a)

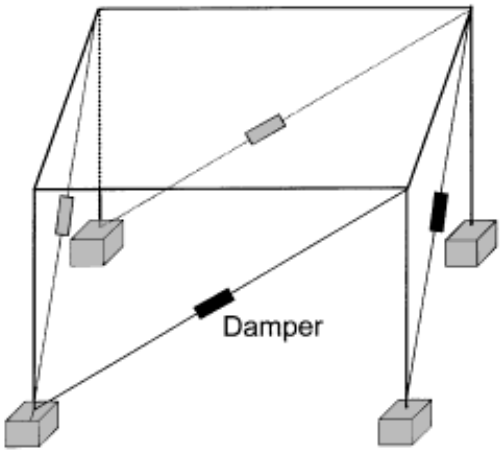

(b)

Figure 1. One-storey system considered: (a) plan, (b) elevation

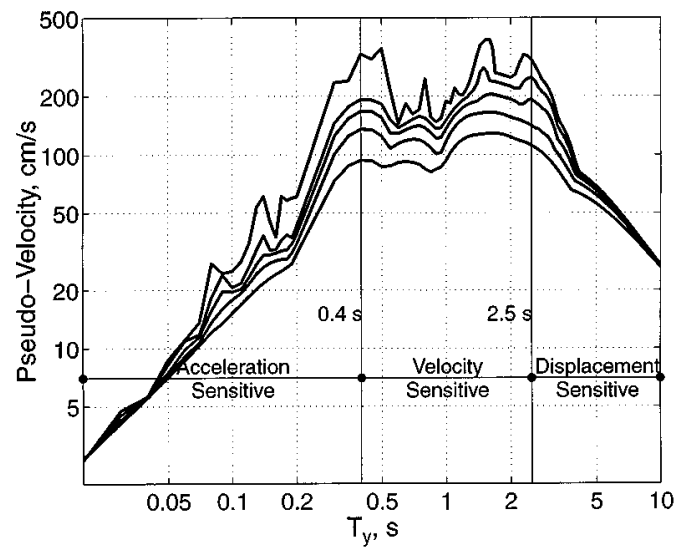

Figure 2. Response spectra for $360^{\circ}$ component of the ground motion recorded at the Sylmar County Hospital during the 1994 Northridge earthquake $; \zeta=0,2,5,10$ and 20 per cent

Figure 2 shows linear elastic response spectra $(\zeta=0,2,5,10$ and $20 \%)$ for the selected ground motion and approximately identifies various spectral regions: acceleration sensitive region $\left(T_{y}<0.4 \mathrm{~s}\right)$, velocity sensitive region $\left(0.4 \mathrm{~s}<T_{y}<2.5 \mathrm{~s}\right)$, and displacement sensitive region $\left(T_{y}>2.5 \mathrm{~s}\right)$; the procedure used to identify these regions is described elsewhere. ${ }^{23}$ It is apparent from Figure 2 that damping is most effective at reducing the system response in the velocity sensitive region of the spectrum. This effect spills over into adjoining portions of the acceleration sensitive region $\left(0.05 \mathrm{~s} \leqslant T_{y} \leqslant 0.4 \mathrm{~s}\right)$ and the displacement sensitive region $\left(2.5 \mathrm{~s} \leqslant T_{y} \leqslant 3 \mathrm{~s}\right)$. This indicates that it would be sufficient to investigate earthquake response of systems with periods in the range of $0.05 \mathrm{~s} \leqslant T_{y} \leqslant 3 \mathrm{~s}$ for the purpose of evaluating the effects of damping.

In general, details of earthquake response may vary from one ground motion to the next. However, a single ground motion was considered to be appropriate for this exploratory study because it is well established that overall response trends identified from various regions of response spectrum for one earthquake are generally valid for the corresponding spectral regions of other ground motions. ${ }^{23}$ 


\section{EQUATIONS OF MOTION}

The one-way symmetric system (Figure 1) has two degrees of freedom (DOF) when subjected to ground motion along the $Y$-axis: translation along the $Y$-axis and rotation about a vertical axis. The displacement vector $\mathbf{u}$ for the system is defined by $\mathbf{u}^{\mathrm{T}}=\left\langle u_{y} a u_{\theta}\right\rangle$ where $u_{y}$ is the horizontal displacement relative to the ground of the CM along the $Y$-axis, $u_{\theta}$ is the rotation of the deck about a vertical axis, and $a$ is the plan dimension of the system along the $X$-axis.

Let $m$ represent the total mass of the deck and let $\rho$ denote the mass radius of gyration. Then the mass matrix of the system with respect to the DOF $\mathbf{u}$ is given by

$$
M=\left[\begin{array}{cc}
m & 0 \\
0 & \left(\frac{\rho}{a}\right)^{2} m
\end{array}\right]
$$

The $\rho$ for a deck with mass uniformly distributed in its plan is given as

$$
\rho=\sqrt{\frac{a^{2}+d^{2}}{12}}=a \sqrt{\frac{1+\alpha^{2}}{12 \alpha^{2}}}
$$

where $\alpha=a / d$ is the aspect ratio of the deck. Then the mass matrix can be expressed as

$$
M=\left[\begin{array}{cc}
m & 0 \\
0 & \frac{1+\alpha^{2}}{12 \alpha^{2}} m
\end{array}\right]
$$

Let $K_{x i}$ represent the lateral stiffness and $y_{i}$ the distance from the CM of the $i$ th resisting element along the $X$-axis; let $K_{y i}$ denote the lateral stiffness and $x_{i}$ the distance from the $\mathrm{CM}$ of the $i$ th resisting element along the $Y$-axis. Then

$$
K_{y}=\sum_{i} K_{y i}, \quad \text { and } \quad K_{\theta}=\sum_{i} K_{x i} y_{i}^{2}+\sum_{i} K_{y i} x_{i}^{2}
$$

are the translational stiffness of the system along the $Y$-axis and the torsional stiffness of the system about a vertical axis at the $\mathrm{CM}$, respectively. Location of the $\mathrm{CR}$ from the $\mathrm{CM}$ is defined by the stiffness eccentricity, $e$, as

$$
e=\frac{1}{K_{y}} \sum_{i} x_{i} K_{y i}
$$

It can easily be shown ${ }^{1}$ that

$$
K_{\theta}=e^{2} K_{y}+K_{\theta R}
$$

in which $K_{\theta R}$ is the torsional stiffness of the system about a vertical axis at the CR. The stiffness matrix of the system can now be expressed as

$$
\mathbf{K}=\left[\begin{array}{cc}
K_{y} & \frac{e}{a} K_{y} \\
\frac{e}{a} K_{y} & \left(\frac{e}{a}\right)^{2} K_{y}+\frac{1}{a^{2}} K_{\theta R}
\end{array}\right]
$$


With both mass and stiffness matrices known, the stiffness-and-mass-proportional damping matrix of the system can be determined as

$$
\mathbf{C}=a_{0} \mathbf{M}+a_{1} \mathbf{K}
$$

in which constants $a_{0}$ and $a_{1}$ depend on damping ratios, $\zeta_{1}$ and $\zeta_{2}$, in the two vibration modes of the system. The damping matrix of equation (8) accounts for the natural capacity of the system to dissipate energy.

Let $C_{x i}$ represent the velocity-proportional damping coefficient and $y_{i}$ the distance from the CM of the $i$ th fluid viscous damper (FVD) along the $X$-axis; let $C_{y i}$ denote the damping coefficient and $x_{i}$ the distance from the $\mathrm{CM}$ of the $i$ th FVD along the $Y$-axis. Then

$$
C_{y}=\sum_{i} C_{y i}, \quad \text { and } \quad C_{\theta}=\sum_{i} C_{x i} y_{i}^{2}+\sum_{i} C_{y i} x_{i}^{2}
$$

are the translational damping coefficient of the system along the $Y$-axis and the torsional damping coefficient of the system about a vertical axis at the CM, respectively. Location of the CSD from the CM is defined by the supplemental damping eccentricity, $e_{\text {sd }}$, as

$$
e_{\mathrm{sd}}=\frac{1}{C_{y}} \sum_{i} x_{i} C_{y i}
$$

Using a transformation similar to that in equation (6), $C_{\theta}$ can be expressed as

$$
C_{\theta}=e_{\mathrm{sd}}^{2} C_{y}+C_{\theta \mathrm{sd}}
$$

in which $C_{\theta \mathrm{sd}}$ is the torsional damping coefficient of the system about a vertical axis at the CSD. The damping matrix of the system due to FVDs can now be expressed as

$$
\mathbf{C}_{\mathrm{sd}}=\left[\begin{array}{cc}
C_{y} & \frac{e_{\mathrm{sd}}}{a} C_{y} \\
\frac{e_{\mathrm{sd}}}{a} C_{y} & \left(\frac{e_{\mathrm{sd}}}{a}\right)^{2} C_{y}+\frac{1}{a^{2}} C_{\theta \mathrm{sd}}
\end{array}\right]
$$

Let us define the supplemental damping radius of gyration, $\rho_{\mathrm{sd}}$, as

$$
\rho_{\mathrm{sd}}=\sqrt{\frac{C_{\theta \mathrm{sd}}}{C_{y}}}
$$

The damping matrix of equation (12) can then be expressed as

$$
\mathbf{C}_{\mathrm{sd}}=\left[\begin{array}{cc}
C_{y} & \frac{e_{\mathrm{sd}}}{a} C_{y} \\
\frac{e_{\mathrm{sd}}}{a} C_{y} & \left\{\left(\frac{e_{\mathrm{sd}}}{a}\right)^{2}+\left(\frac{\rho_{\mathrm{sd}}}{a}\right)^{2}\right\} C_{y}
\end{array}\right]
$$


The equations of motion for the one-way symmetric system (Figure 1) subjected to ground acceleration $\ddot{u}_{\mathrm{g}}$ along the $Y$-axis are

$$
\begin{gathered}
{\left[\begin{array}{cc}
m & 0 \\
0 & m \frac{1+\alpha^{2}}{12 \alpha^{2}}
\end{array}\right]\left\{\begin{array}{c}
\ddot{u}_{y} \\
a \ddot{u}_{\theta}
\end{array}\right\}+\left(a_{0}\left[\begin{array}{cc}
m & 0 \\
0 & m \frac{1+\alpha^{2}}{12 \alpha^{2}}
\end{array}\right]+a_{1}\left[\begin{array}{cc}
K_{y} & \frac{e}{a} K_{y} \\
\frac{e}{a} K_{y} & \left(\frac{e}{a}\right)^{2} K_{y}+\frac{1}{a^{2}} K_{\theta R}
\end{array}\right]\right)\left\{\begin{array}{c}
\dot{u}_{y} \\
a \dot{u}_{\theta}
\end{array}\right\}} \\
+\left[\begin{array}{c}
C_{y} \\
\frac{e_{\mathrm{sd}}}{a} C_{y}\left\{\left(\frac{e_{\mathrm{sd}}}{a}\right)^{2}+\left(\frac{\rho_{\mathrm{sd}}}{a}\right)^{2}\right\}
\end{array}\right]\left\{\begin{array}{c}
\dot{u}_{y} \\
a \dot{u}_{\theta}
\end{array}\right\}+\left[\begin{array}{cc}
K_{y} & \frac{e}{a} K_{y} \\
\frac{e}{a} K_{y} & \left(\frac{e}{a}\right)^{2} K_{y}+\frac{1}{a^{2}} K_{\theta R}
\end{array}\right]\left\{\begin{array}{c}
u_{y} \\
a u_{\theta}
\end{array}\right\}=-\left\{\begin{array}{c}
m \\
0
\end{array}\right\} \ddot{u}_{\mathrm{g}}
\end{gathered}
$$

After dividing by $m$ and some algebraic manipulations, equation (15) leads to

$$
\begin{aligned}
& {\left[\begin{array}{cc}
1 & 0 \\
0 & \frac{1+\alpha^{2}}{12 \alpha^{2}}
\end{array}\right]\left\{\begin{array}{c}
\ddot{u}_{y} \\
a \ddot{u}_{\theta}
\end{array}\right\}+\left(a_{0}\left[\begin{array}{cc}
1 & 0 \\
0 & \frac{1+\alpha^{2}}{12 \alpha^{2}}
\end{array}\right]+a_{1} \omega_{y}^{2}\left[\begin{array}{cc}
1 & \bar{e} \\
\bar{e} & \bar{e}^{2}+\frac{1+\alpha^{2}}{12 \alpha^{2}} \Omega_{\theta}^{2}
\end{array}\right]\right)\left\{\begin{array}{c}
\dot{u}_{y} \\
a \dot{u}_{\theta}
\end{array}\right\}} \\
& +2 \omega_{y} \zeta_{\mathrm{sd}}\left[\begin{array}{cc}
1 & \bar{e}_{\mathrm{sd}} \\
\bar{e}_{\mathrm{sd}} & \bar{e}_{\mathrm{sd}}^{2}+\bar{\rho}_{\mathrm{sd}}^{2}
\end{array}\right]\left\{\begin{array}{c}
\dot{u}_{y} \\
a \dot{u}_{\theta}
\end{array}\right\}+\omega_{y}^{2}\left[\begin{array}{cc}
1 & \bar{e} \\
\bar{e} & \bar{e}^{2}+\frac{1+\alpha^{2}}{12 \alpha^{2}} \Omega_{\theta}^{2}
\end{array}\right]\left\{\begin{array}{c}
u_{y} \\
a u_{\theta}
\end{array}\right\}=-\left\{\begin{array}{l}
1 \\
0
\end{array}\right\} \ddot{u}_{\mathrm{g}}
\end{aligned}
$$

in which $\omega_{y}=\sqrt{K_{y} / m}=$ transverse vibration frequency; $\Omega_{\theta}=\omega_{\theta} / \omega_{y}=$ ratio of the torsional and transverse frequencies with $\omega_{\theta}=\sqrt{K_{\theta R} / m \rho^{2}}=$ torsional frequency; $\zeta_{\text {sd }}=C_{y} / 2 m \omega_{y}=$ supplemental damping ratio; $\bar{e}=e \div a=$ normalized stiffness eccentricity; $\bar{e}_{\mathrm{sd}}=e_{\mathrm{sd}} \div a=$ normalized supplemental damping eccentricity; and $\bar{\rho}_{\mathrm{sd}}=\rho_{\mathrm{sd}} \div a=$ normalized supplemental damping radius of gyration.

\section{CONTROLLING SYSTEM PARAMETERS}

Equation (16) indicates that the linear elastic response of one-storey, asymmetric-plan systems with FVDs depends on two sets of parameters. The first set of parameters corresponding to the system without FVDs consists of (1) transverse vibration period, $T_{y}=2 \pi / \omega_{y}\left(\omega_{y}=\right.$ transverse vibration frequency) of the corresponding symmetric-plan system; (2) normalized stiffness eccentricity, $\bar{e}$; (3) ratio of the torsional and transverse frequencies, $\Omega_{\theta} ;$ (4) aspect ratio, $\alpha$; and (5) mass and stiffness proportional constants, $a_{0}$ and $a_{1}$, which in turn depend on the natural damping ratios, $\zeta_{1}$ and $\zeta_{2}$, in the two vibration modes of the system. The aspect ratio was included as one of the system parameters because it facilitated a more appealing definition of the stiffness eccentricity as a percentage (or fraction) of the plan dimension. If the stiffness eccentricity were normalized by the mass radius of gyration, the aspect ratio would not be an independent system parameter. Since physical interpretation of many of these parameters has been discussed in an earlier publication, ${ }^{1}$ they are not described in detail here.

The second set of parameters corresponding to supplemental damping consists of (1) supplemental damping ratio, $\zeta_{\mathrm{sd}} ;(2)$ normalized supplemental damping eccentricity, $\bar{e}_{\mathrm{sd}}$; and (3) normalized supplemental damping radius of gyration, $\bar{\rho}_{\text {sd }}$. $\zeta_{\text {sd }}$ is indicative of the amount of additional damping, as a fraction of the critical value, provided by FVDs in the transverse vibration mode of the corresponding symmetric-plan system; and $\bar{e}_{\text {sd }}$ is indicative of how unevenly FVDs are located within the system plan. A zero value of $\bar{e}_{\text {sd }}$ implies that FVDs are located symmetrically about the CM, whereas non-zero values $\left(\bar{e}_{\mathrm{sd}}\right.$ can take on 
a positive as well as a negative value) indicate uneven distribution. Since FVDs cannot be located outside the system plan, the limiting values of $\bar{e}_{\mathrm{sd}}$ are -0.5 and 0.5 ; these values correspond to all dampers located either on the flexible edge or on the stiff edge (Figure 1). $\bar{\rho}_{\text {sd }}$ indicates how much farther apart from the CSD the FVDs are located. This parameter is also indicative of the damping in the torsional mode of vibration of the corresponding symmetric-plan system. Zero value of $\bar{\rho}_{\text {sd }}$ implies that all FVDs are located at the CSD and that they provide zero damping in the torsional mode, whereas larger values indicate that FVDs are located farther from the CSD and that damping is increased in the torsional mode.

It should be noted that the system damping was specified as sum of (1) the stiffness and mass proportional classical damping depending on $\zeta_{1}$ and $\zeta_{2}$; and (2) the supplemental damping due to the FVDs characterized by $\zeta_{\text {sd }}, \bar{e}_{\mathrm{sd}}$ and $\bar{\rho}_{\mathrm{sd}}$. Clearly, systems without FVDs would possess only classical damping. Also note that the locations of dampers in this investigation were not necessarily restricted to system edges; they are shown to be on edges only for the purpose of clarity (Figure 1(b)).

The damping coefficient of an FVD, in general, depends on the frequency and amplitude of motion as well as on the operating temperature. ${ }^{8}$ Furthermore, FVD may also exhibit some viscoelastic force-deformation behaviour, i.e., the force in an FVD depends not only on the velocity but also on the displacement. However, due to exploratory nature of this investigation, these parameters were not considered; they will be included at a later stage.

\section{SELECTED SYSTEM PARAMETERS}

Responses are presented for the following values of system parameters. Values of $T_{y}$ were selected in the range of $0.05-3 \mathrm{~s}$ to represent many low-rise and mid-rise buildings for which supplemental damping is expected to significantly influence the response. The selected value of $\Omega_{\theta}=1$ represents systems with strong coupling between lateral and torsional motions in the elastic range. In order to investigate how the effects of supplemental damping differ for torsionally-very-flexible and torsionally-very-stiff systems, values of $\Omega_{\theta}=0.5$ and 2.0 were also considered. The normalized stiffness eccentricity $\bar{e}$ was selected as 0.2 which represents an eccentricity of 20 per cent of the plan dimension. The aspect ratios, $\alpha$, of the selected systems were fixed at two. The constants $a_{0}$ and $a_{1}$ in equation (8) were selected such that damping ratios in both vibration modes of the system with $\bar{e}$ fixed were equal to 5 per cent, i.e., $\zeta_{1}=\zeta_{2}=\zeta=5$ per cent.

The value of $\zeta_{\text {sd }}$ was fixed at 10 per cent for most cases; for a limited number of cases, however, variations of $\zeta_{\mathrm{sd}}$ in the range of $0-50$ per cent were considered. In general, three values of $\bar{e}_{\mathrm{sd}}=0 \cdot 2,0$ and $-0 \cdot 2$ were selected. The first corresponds to the supplemental damping eccentricity equal to and in the same direction as the selected stiffness eccentricity, i.e., coincidental locations of the CR and CSD. The second value corresponds to even distribution of FVDs about the CM and thus the identical location of the CM and CSD. The last value corresponds to equal values of the two eccentricities, but with the CSD located on the opposite side of the CM from the CR. For selected cases, variations of $\bar{e}_{\mathrm{sd}}$ in the range of $-0.5-0.5$ were also considered. The selected values of $\bar{\rho}_{\text {sd }}=0,0.2$ and 0.5 represent low, medium, and large spread of FVDs about the CSD.

\section{RESPONSE QUANTITIES}

The response quantities of interest were the peak deformations $u_{\mathrm{f}}$ and $u_{\mathrm{s}}$ at the flexible and the stiff edge, respectively, of the system. If the system plan were symmetric, these deformations would be identical, i.e., $u_{\mathrm{f}}=u_{\mathrm{s}}=u_{\mathrm{o}}$. The deviations in $u_{\mathrm{f}}$ and $u_{\mathrm{s}}$ from $u_{\mathrm{o}}$ are indicative of the effects of plan asymmetry. Therefore, the response quantities selected in this investigation were the deformations of the flexible and stiff edges in asymmetric-plan system normalized by the deformation of the corresponding symmetric-plan system, $\bar{u}_{\mathrm{f}}=u_{\mathrm{f}} \div u_{\mathrm{o}}$ and $\bar{u}_{\mathrm{s}}=u_{\mathrm{s}} \div u_{\mathrm{o}}$. A value of the normalized edge deformation by more than one indicates a larger edge deformation in the asymmetric-plan system as compared to the corresponding symmetric-plan system; conversely, a value of normalized edge deformation smaller than one implies a smaller edge 
deformation in the asymmetric-plan system. It is useful to note that no FVDs were included in the corresponding symmetric-plan system; the system possessed only classical damping with $\zeta_{1}=\zeta_{2}=\zeta=5 \%$.

The peak values of edge deformations were selected to be the response quantities of interest, rather than peak values of translational and rotational displacements at $\mathrm{CM}$ or $\mathrm{CR},{ }^{1}$ because they are indicative of (1) deformation demands on outermost lateral load resisting elements of the system, (2) deformation demands on the cladding system, which generally consists of brittle, non-ductile elements sensitive to excessive deformations, and (3) clear spacing required between adjacent buildings in order to avoid pounding. Peak values of the translational and rotational deformations at CM (or CR) may occur at different times and cannot be combined directly to obtain deformation demands at some other location on the system plan.

\section{EFFECTS OF PLAN ASYMMETRY}

Although the effects of plan asymmetry in linearly elastic systems without supplemental damping have been extensively investigated, ${ }^{1}$ it is useful to summarize them here prior to a detailed examination of the effects of supplemental damping. For this purpose, the normalized deformations at the flexible and stiff edges, $\bar{u}_{\mathrm{f}}$ and $\bar{u}_{\mathrm{s}}$, respectively, of asymmetric-plan systems without FVDs were computed and are presented in Figure 3 for three values of $\Omega_{\theta}=0 \cdot 5,1$ and 2 . Described first are the effects of plan asymmetry in strongly coupled asymmetric-plan systems $\left(\Omega_{\theta}=1\right)$. Subsequently, responses for torsionally-very-flexible $\left(\Omega_{\theta}=0 \cdot 5\right)$ and torsionally-very-rigid $\left(\Omega_{\theta}=2\right)$ systems are compared with those for strongly coupled $\left(\Omega_{\theta}=1\right)$ systems in order to point out how these effects are influenced by $\Omega_{\theta}$.

As expected, the results for strongly coupled systems $\left(\Omega_{\theta}=1\right)$ show that plan asymmetry significantly influences edge deformations. In particular, deformations of the flexible edge may be much larger in asymmetric-plan systems as compared to the corresponding symmetric-plan system (Figure 3(a)). For many asymmetric-plan systems, this deformation may exceed the deformation of the same edge in the corresponding symmetric-plan system by a factor of over two. The deformations of the stiff edge, on the other hand, are smaller in asymmetric-plan systems as compared to the corresponding symmetric-plan systems (Figure 3(b)). These effects of the plan asymmetry tend to be more pronounced for short-period systems.

The effects of plan asymmetry depend significantly on $\Omega_{\theta}$. For torsionally-very-stiff systems $\left(\Omega_{\theta}=2\right)$, the flexible edge deformations are only slightly larger and the stiff edge deformations slightly smaller as compared to deformations of the corresponding symmetric-plan systems. Furthermore, the effects of plan

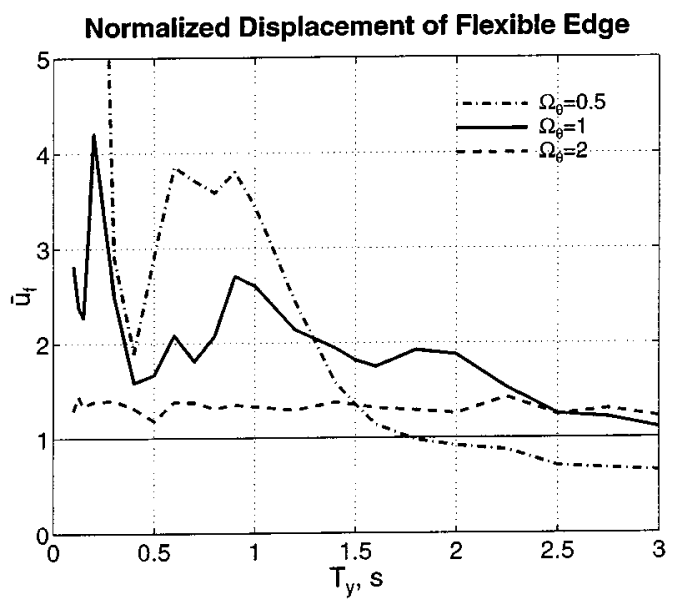

(a)

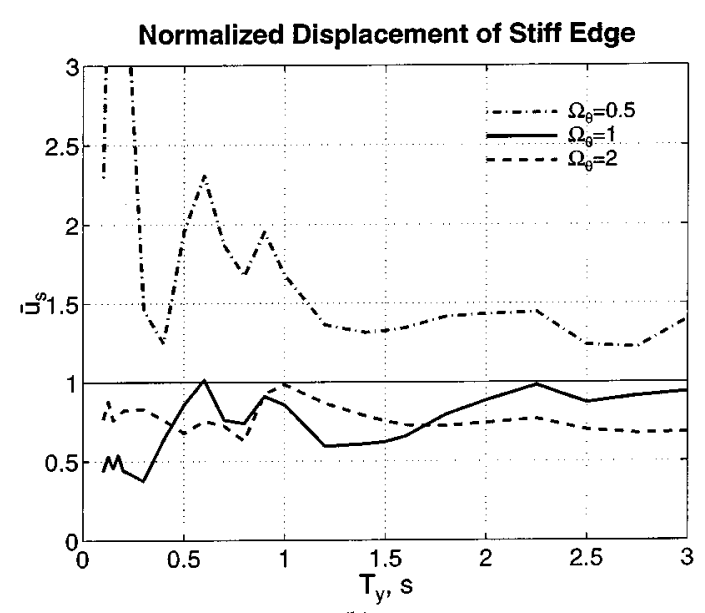

(b)

Figure 3. Normalized edge deformations in asymmetric-plan systems $(\bar{e}=0 \cdot 2 ; \alpha=2 ; \zeta=5$ per cent $)$ 
asymmetry are essentially independent of the system period. For torsionally-very-flexible systems $\left(\Omega_{\theta}=0 \cdot 5\right)$, however, deformations at both edges may be significantly larger as compared to the corresponding symmetric-plan systems, and these effects show strong dependence on the system period. These observations are consistent with previous findings. ${ }^{1}$

It becomes apparent from the summary presented in this section that brittle, non-ductile elements (structural or non-structural), which are sensitive to excessive deformations, when located near the edge may experience much larger damage in asymmetric-plan systems as compared to the same elements in the corresponding symmetric-plan systems. This may occur primarily for elements near the flexible edge of asymmetric-plan systems; for torsionally-very-flexible systems $\left(\Omega_{\theta}=0 \cdot 5\right)$, elements located near the stiff edge may also be vulnerable.

\section{EFFECTS OF SUPPLEMENTAL DAMPING}

Effects of various system parameters related to the supplemental damping $-\bar{e}_{\mathrm{sd}}, \bar{\rho}_{\mathrm{sd}}$, and $\zeta_{\mathrm{sd}}-$ are evaluated by comparing the normalized edge deformations, $\bar{u}_{\mathrm{f}}$ and $\bar{u}_{\mathrm{s}}$, of systems with supplemental dampers with those of systems without supplemental dampers; the later is denoted as the $\zeta_{\text {sd }}=0$ case. Following is a detailed discussion of these effects.

\section{Supplemental damping eccentricity}

Presented in Figure 4 are the normalized edge deformations, $\bar{u}_{\mathrm{f}}$, and $\bar{u}_{\mathrm{s}}$, in asymmetric-plan systems against the period $T_{y}$ for three values of $\bar{e}_{\mathrm{sd}}=0 \cdot 2,0$ and $-0 \cdot 2$ along with the results for asymmetric-plan systems without FVDs (denoted as $\zeta_{\text {sd }}=0$ ). These results show that the supplemental damping has the effect of reducing deformations at both edges. However, the degree of reduction depends significantly on the normalized supplemental damping eccentricity, $\bar{e}_{\mathrm{sd}}$. For the flexible edge, $\bar{e}_{\mathrm{sd}}=-0.2$ led to the largest reduction and $\bar{e}_{\mathrm{sd}}=0.2$ resulted in the smallest reduction (Figure 4(a)). These trends are reversed for the stiff edge, for which $\bar{e}_{\mathrm{sd}}=0.2$ led to the largest reduction and $\bar{e}_{\mathrm{sd}}=-0.2$ resulted in the smallest reduction (Figure 4(b)). For both edges, $\bar{e}_{\mathrm{sd}}=0$ led to an intermediate reduction.

In order to further examine the variation of the above-noted effects with $\bar{e}_{\text {sd }}$, the normalized edge deformations were computed for a range of $\bar{e}_{\text {sd }}$ values between -0.5 and 0.5 for systems with $T_{y}=1 \mathrm{~s}$ and

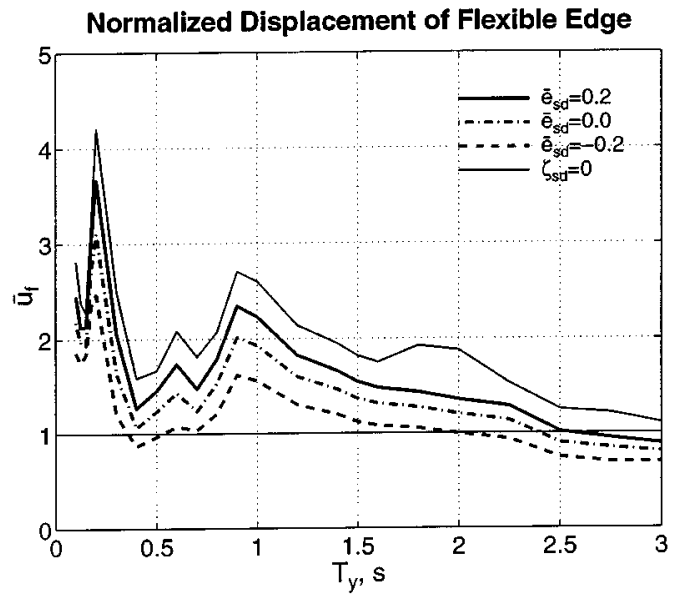

(a)

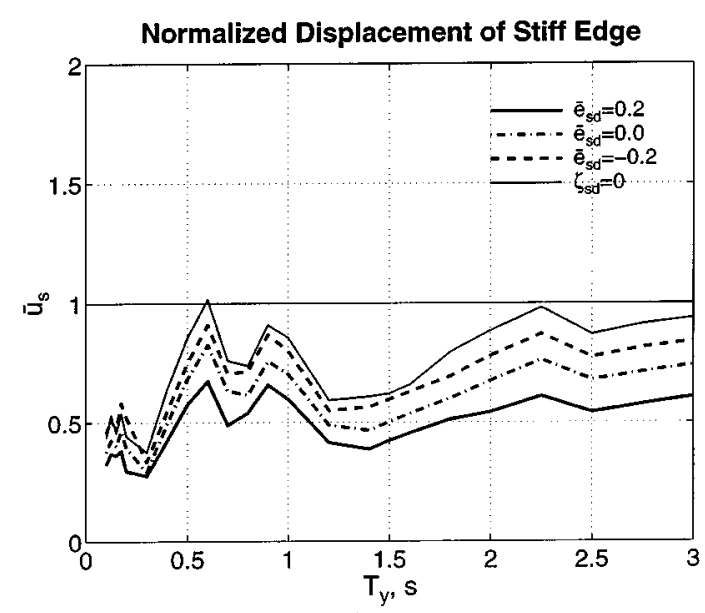

(b)

Figure 4. Normalized edge deformations in asymmetric-plan systems $\left(\bar{e}=0 \cdot 2 ; \Omega_{\theta}=1 ; \alpha=2 ; \zeta=5\right.$ per cent $)$ with supplemental damping $\left(\zeta_{\text {sd }}=10\right.$ per cent and $\left.\bar{\rho}=0 \cdot 2\right)$ and without supplemental damping $\left(\zeta_{\text {sd }}=0\right)$ 


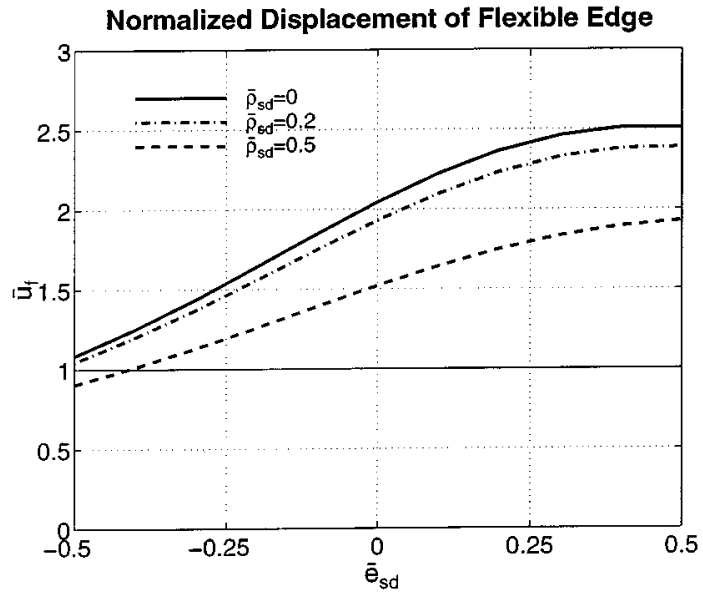

(a)

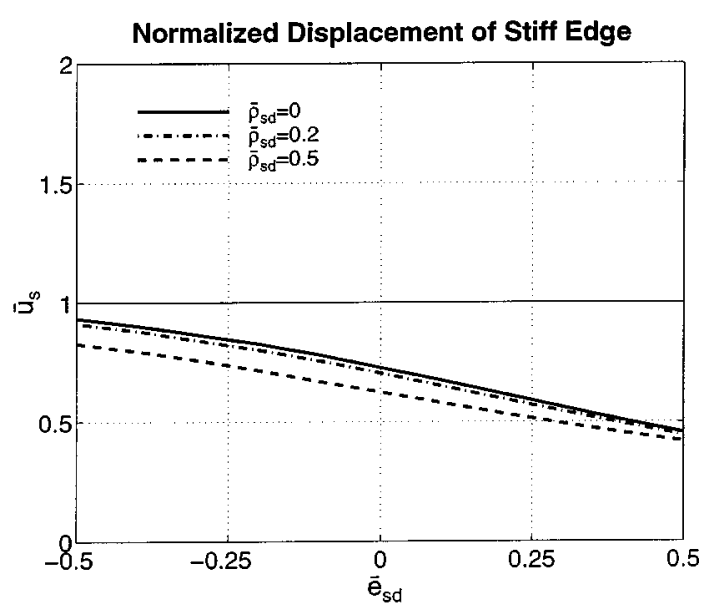

(b)

Figure 5. Normalized edge deformations in asymmetric-plan systems $\left(\bar{e}=0 \cdot 2 ; \Omega_{\theta}=1 ; \alpha=2 ; \zeta=5\right.$ per cent; $\left.T_{y}=1 \mathrm{~s}\right)$ with supplemental damping $\left(\zeta_{\text {sd }}=10\right.$ per cent $)$

are presented in Figure 5; the extreme values of $\bar{e}_{\mathrm{sd}}=-0.5$ and 0.5 correspond to all FVDs located either at the flexible or at the stiff edge, respectively. Although the results are presented for three values of $\bar{\rho}_{\text {sd }}=0,0 \cdot 2$ and $0 \cdot 5$, the discussion in this section will focus on systems with $\bar{\rho}_{\mathrm{sd}}=0 \cdot 2$, the value selected for systems considered in Figure 4; results for $\bar{\rho}_{\text {sd }}=0$ and 0.5 will be utilized in the next section, where the effects of $\bar{\rho}_{\text {sd }}$ are discussed. These results show that deformation of the flexible edge decreases and that of the stiff edge increases as $\bar{e}_{\text {sd }}$ decreases from 0.5 to $-0 \cdot 5$, i.e., the CSD moves from the right to the left of the system plan (Figure 1(a)). These results also show that $\bar{u}_{\mathrm{f}}$ is the smallest for $\bar{e}_{\mathrm{sd}}=-0.5$ (Figure 5(a)), indicating that the largest reduction in deformation of the flexible edge would be obtained by concentrating all FVDs at the flexible edge. A reduction by a factor of nearly two, when compared to the symmetric distribution $\left(\bar{e}_{\mathrm{sd}}=0\right)$, can be achieved for the selected system. The stiff edge deformation, on the other hand, is the smallest for $\bar{e}_{\mathrm{sd}}=5 \cdot 0$, implying that the largest reduction would be obtained by locating all FVDs at the stiff edge (Figure $5(\mathrm{~b}))$. The reduction for this edge by a factor one-and-a-half compared to the symmetric distribution $\left(\bar{e}_{\mathrm{sd}}=0\right)$, while smaller than that for the flexible edge, it is still significant.

Since the effects of plan asymmetry depend significantly on the uncoupled lateral-to-torsional frequency ratio, $\Omega_{\theta}$, results were also generated for torsionally-very-flexible $\left(\Omega_{\theta}=0 \cdot 5\right)$ and torsionally-very-stiff $\left(\Omega_{\theta}=2 \cdot 0\right)$ systems and are presented in Figures 6 and 7. It is apparent from these figures that the effects of supplemental damping and damping eccentricity tend to be more pronounced on the flexible edge of torsionally-very-flexible systems $\left(\Omega_{\theta}=0 \cdot 5\right)$ as compared to strongly coupled systems $\left(\Omega_{\theta}=1\right)$ (Figures $6($ a) and 4(a)). In particular, providing supplemental damping (curve for $\zeta_{\text {sd }}=0$ compared to other curves) and a changes in $\bar{e}_{\text {sd }}$ from 0.2 to -0.2 (curve for various values of $\bar{e}_{\text {sd }}$ ) resulted in a much larger reduction in the flexible edge deformations of torsionally-very-flexible systems. This effect is more pronounced for systems with $T_{y}=0.5-1.5 \mathrm{~s}$. As noted previously (Figure 3(b)), the stiff edge deformations of torsionally-very-flexible systems may exceed those in the corresponding symmetric-plan systems. The supplemental damping reduces the deformation of this edge as well; however, this reduction is not very sensitive to $\bar{e}_{\text {sd }}$ (Figure 6(b)).

While supplemental damping reduces edge deformations in torsionally-very-stiff systems $\left(\Omega_{\theta}=2\right)$, the reductions tend to be smaller than those for systems with $\Omega_{\theta}=1$ (Figures 7 and 4). Furthermore, these reductions are not very sensitive to $\bar{e}_{\text {sd }}$, as is apparent from nearly identical curves for $\bar{e}_{\text {sd }}=0 \cdot 2,0$ and $-0 \cdot 2$ (Figure 7). 


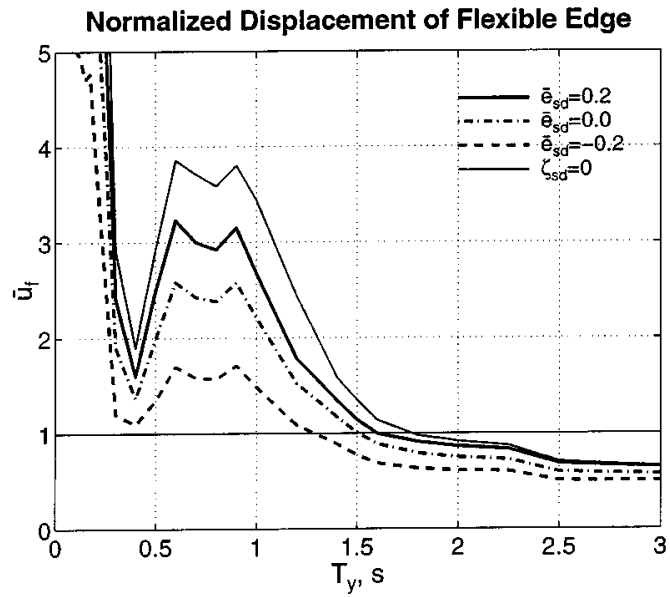

(a)

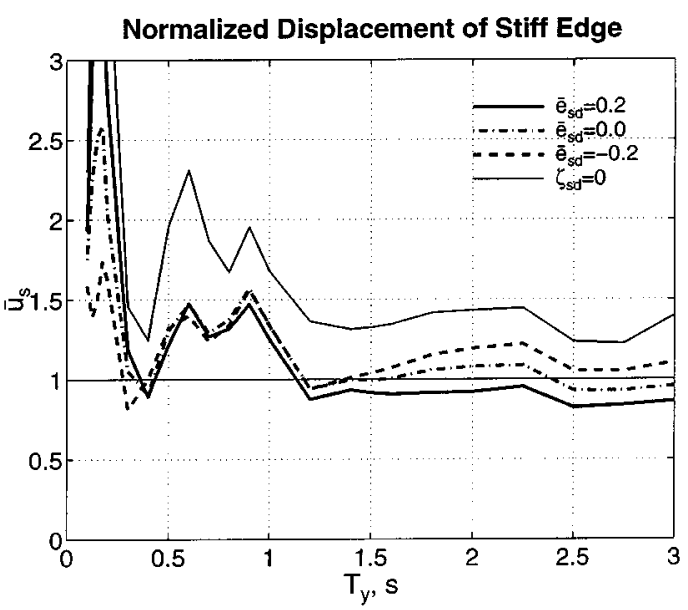

(b)

Figure 6. Normalized edge deformations in asymmetric-plan systems $\left(\bar{e}=0 \cdot 2 ; \Omega_{\theta}=0 \cdot 5 ; \alpha=2 ; \zeta=5\right.$ per cent $)$ with supplemental damping $\left(\zeta_{\text {sd }}=10\right.$ per cent and $\left.\bar{\rho}_{\text {sd }}=0 \cdot 2\right)$ and without supplemental damping $\left(\zeta_{\text {sd }}=0\right)$

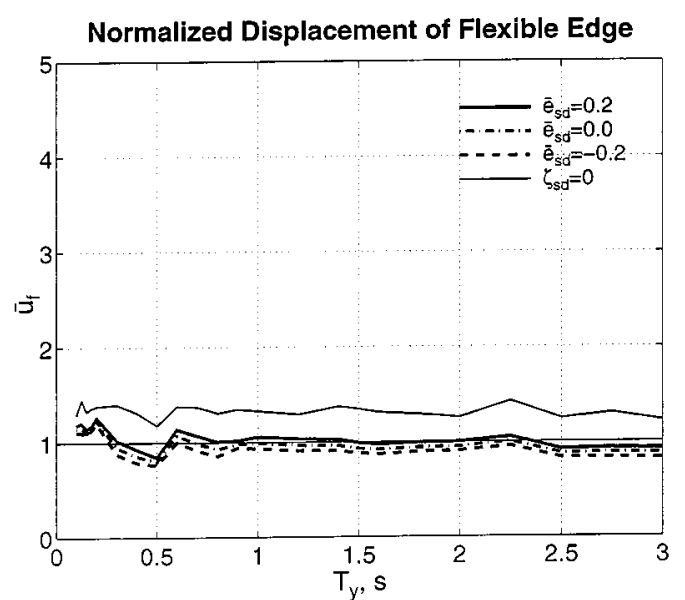

(a)

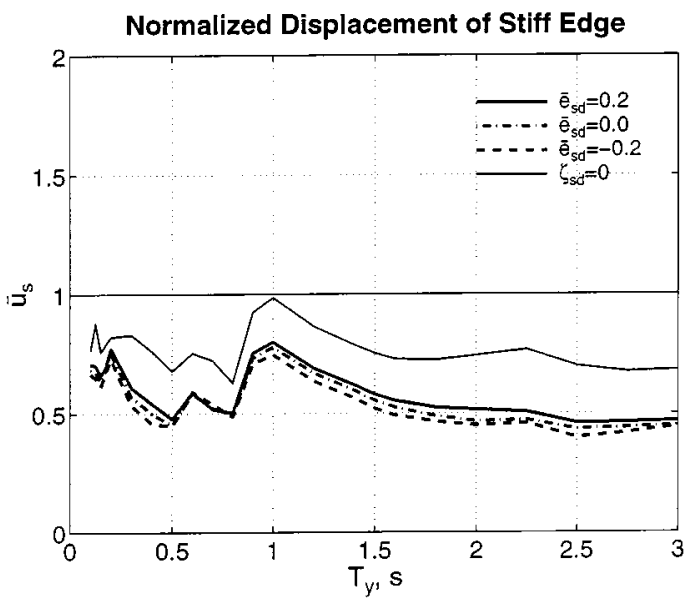

(b)

Figure 7. Normalized edge deformations in asymmetric-plan systems $\left(\bar{e}=0 \cdot 2 ; \Omega_{\theta}=2 ; \alpha=2 ; \zeta=5\right.$ per cent $)$ with supplemental damping $\left(\zeta_{\mathrm{sd}}=10\right.$ per cent and $\left.\bar{\rho}_{\mathrm{sd}}=0 \cdot 2\right)$ and without supplemental damping $\left(\zeta_{\mathrm{sd}}=0\right)$

The results presented so far clearly show the importance of a plan-wise distribution of FVDs for controlling edge deformations in asymmetric-plan systems. While a plan-wise uniform (or symmetrical) distribution of FVDs, i.e., $\bar{e}_{\text {sd }}=0$, led to a reduction in edge deformations when compared to systems without FVDs $\left(\zeta_{s d}=0\right)$, it did not result in the largest possible reduction. In particular, deformations of the flexible edge are most reduced by distributing FVDs such that the CSD is on the opposite side of the CM from the $\mathrm{CR}$, whereas deformations of the stiff edge are most reduced by distributing FVDs such that the CSD is on the same side of the $\mathrm{CM}$ as the CR. In either case, an additional reduction, compared to the symmetric distribution $\left(\bar{e}_{\mathrm{sd}}=0\right)$ of FVDs, by a factor of up to two may be achieved by appropriately selecting the CSD location; the total reduction may be by a factor of up to three when compared to systems without FVDs $\left(\zeta_{\mathrm{sd}}=0\right)$. 


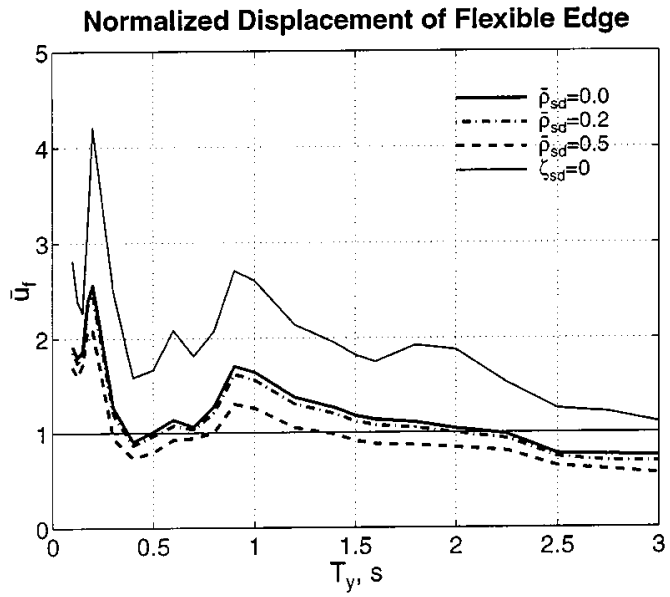

(a)

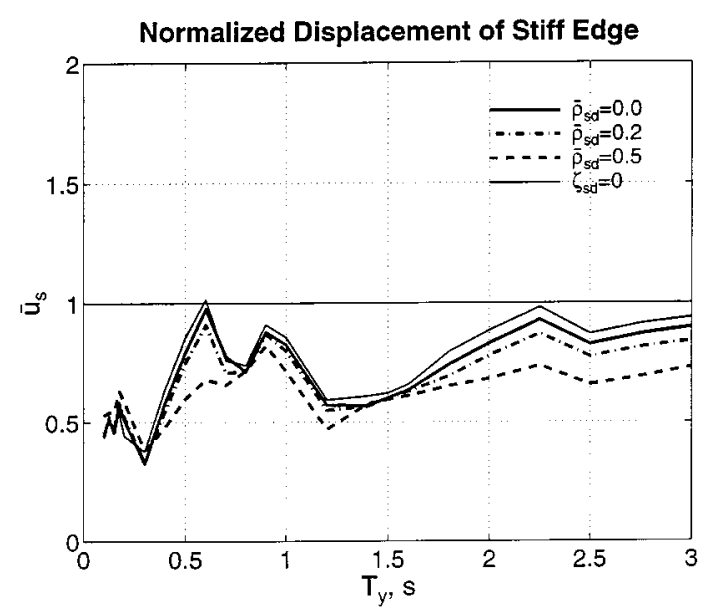

(b)

Figure 8. Normalized edge deformations in asymmetric-plan systems $\left(\bar{e}=0 \cdot 2 ; \Omega_{\theta}=1 ; \alpha=2 ; \zeta=5\right.$ per cent $)$ with supplemental damping $\left(\zeta_{\mathrm{sd}}=10\right.$ per cent and $\left.\bar{e}_{\mathrm{sd}}=-0 \cdot 2\right)$ and without supplemental damping $\left(\zeta_{\mathrm{sd}}=0\right)$

It is also apparent that the same distribution of FVDs does not lead to the most reduction in deformations of both edges: the distribution that results in the largest reduction in the flexible edge deformation leads to the smallest reduction in the stiff edge deformation and vice versa. For asymmetric-plan systems, the flexible edge is generally the most critical edge because of higher earthquake-induced deformations. Therefore, FVDs should be distributed such that the CSD is as far away from the CM, on the side opposite to the CR, as physically possible - a distribution that leads to the largest reduction in deformation of the flexible edge. Although this distribution does not lead to the largest possible reduction in deformation of the stiff edge, it nonetheless leads to a reduction in deformations of this edge as compared to deformations of the same edge is systems without FVDs.

\section{Supplemental damping radius of gyration}

Presented in Figures 8-10 are the normalized deformations, $\bar{u}_{\mathrm{f}}$ and $\bar{u}_{\mathrm{s}}$, in asymmetric-plan systems against the period $T_{y}$ for three values of $\bar{\rho}_{\mathrm{sd}}=0,0.2$ and 0.5 , along with the results for asymmetric-plan systems without FVDs $\left(\zeta_{\text {sd }}=0\right)$. The first value of $\bar{\rho}_{\text {sd }}$ corresponds to all FVDs located at the CSD, implying no spread in the supplemental damping, whereas the second and the third values correspond to moderate and large spreads in the supplemental damping from the CSD. The presented results are for a fixed value of $\bar{e}_{\mathrm{sd}}=-0 \cdot 2$.

It is apparent from these results that a larger values of $\bar{\rho}_{\text {sd }}$ leads to a larger reduction in edge deformations. This trend applies to deformations at both edges and is also supported by results presented earlier in Figure 5. Furthermore, these effects are the most pronounced for torsionally-very-flexible systems (Figure 9); the edge deformations of torsionally-very-rigid systems are essentially unaffected by $\bar{\rho}_{\text {sd }}$ (Figure 10 ).

The results presented so far indicate that in order to obtain the largest reduction in deformation of the flexible edge, FVDs should be distributed in the system plan such that both $\bar{e}_{\text {sd }}$ and $\bar{\rho}_{\text {sd }}$ take on the largest possible values; the value of $\bar{e}_{\mathrm{sd}}$ should also be negative. However, $\bar{e}_{\mathrm{sd}}$ and $\bar{\rho}_{\mathrm{sd}}$ cannot physically take on the largest possible values simultaneously. For example, the largest possible negative values of $\bar{e}_{\text {sd }}$ occurs when all FVDs are located at the flexible edge; however, $\bar{\rho}_{\text {sd }}$ becomes zero for such a distribution. Therefore, an optimization problem would have to be solved in order to obtain the combination of values for the $\bar{e}_{\text {sd }}$ and $\bar{\rho}_{\text {sd }}$ that results in the largest reduction without violating the physical constraints. However, the following simple guidelines may be used to establish a near-optimal solution. 


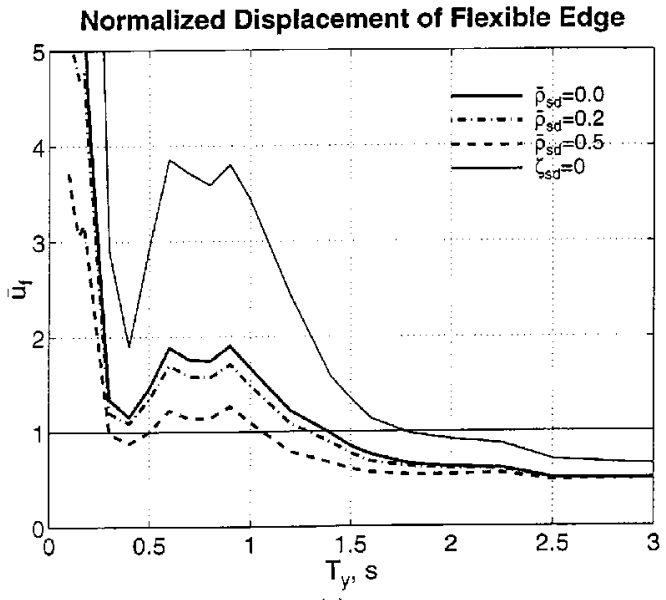

(a)

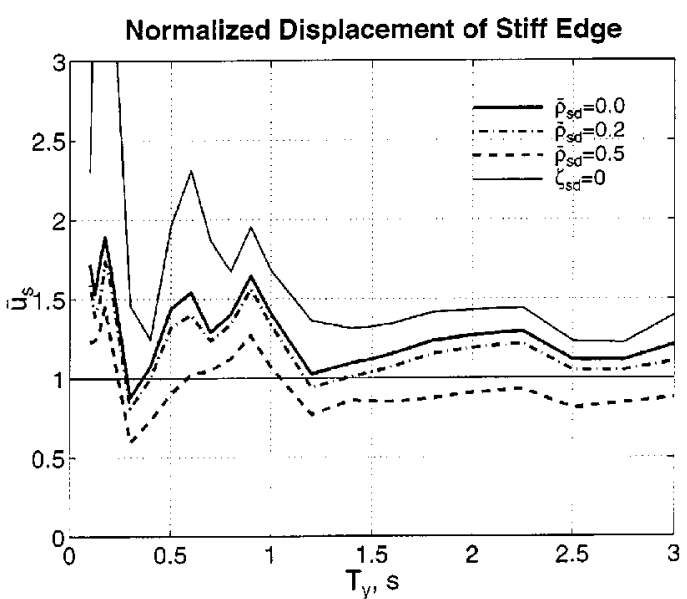

(b)

Figure 9. Normalized edge deformations in asymmetric-plan systems $\left(\bar{e}=0 \cdot 2 ; \Omega_{\theta}=0 \cdot 5 ; \alpha=2 ; \zeta=5\right.$ per cent $)$ with supplemental damping $\left(\zeta_{\mathrm{sd}}=10\right.$ per cent and $\left.\bar{e}_{\mathrm{sd}}=-0 \cdot 2\right)$ and without supplemental damping $\left(\zeta_{\mathrm{sd}}=0\right)$

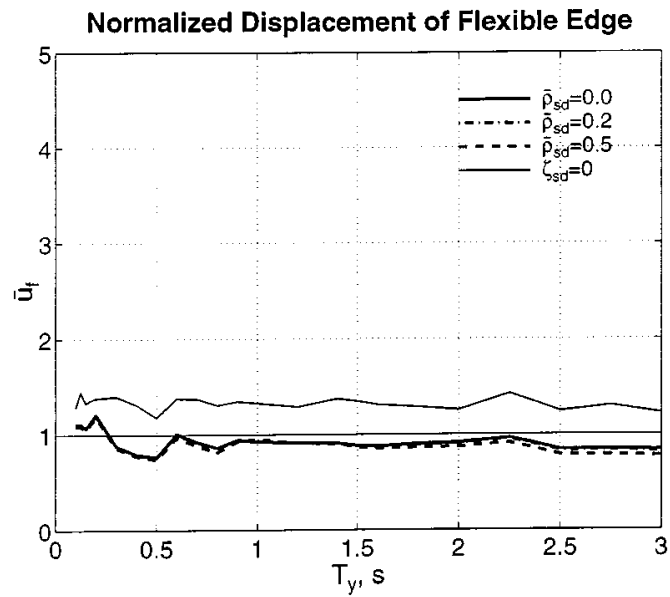

(a)

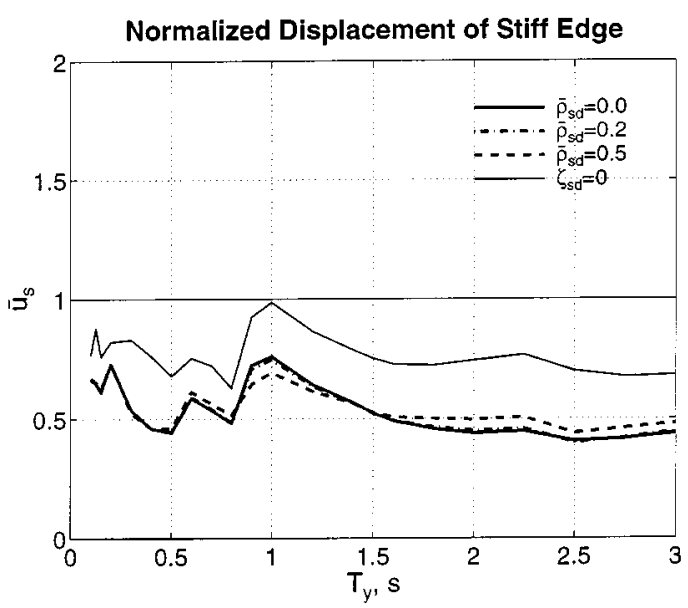

(b)

Figure 10. Normalized edge deformations in asymmetric-plan systems $\left(\bar{e}=0 \cdot 2 ; \Omega_{\theta}=2 ; \alpha=2 ; \zeta=5\right.$ per cent $)$ with supplemental damping $\left(\zeta_{\mathrm{sd}}=10\right.$ per cent and $\left.\bar{e}_{\mathrm{sd}}=-0 \cdot 2\right)$ and without supplemental damping $\left(\zeta_{\mathrm{sd}}=0\right)$

First, as few FVDs as possible should be used in the direction under consideration and the outermost dampers should be located at the two edges. These FVDs should be proportioned such that the damping eccentricity is nearly equal to the structural eccentricity, but opposite in sign (i.e., CSD is located on the opposite side of the CM from the CR). Although an arrangement with just two FVDs is preferable from the theoretical point of view because it leads to the largest possible value of the $\bar{\rho}_{\text {sd }}$, at least three FVDs should be used in order to provide some redundancy in the system. Second, FVDs should also be included in the perpendicular direction. Although the perpendicular FVDs do not affect $\bar{e}_{\text {sd }}$ (equation (10)), they increase the value $\bar{\rho}_{\text {sd }}$ (equations (9), (11), (13)). 


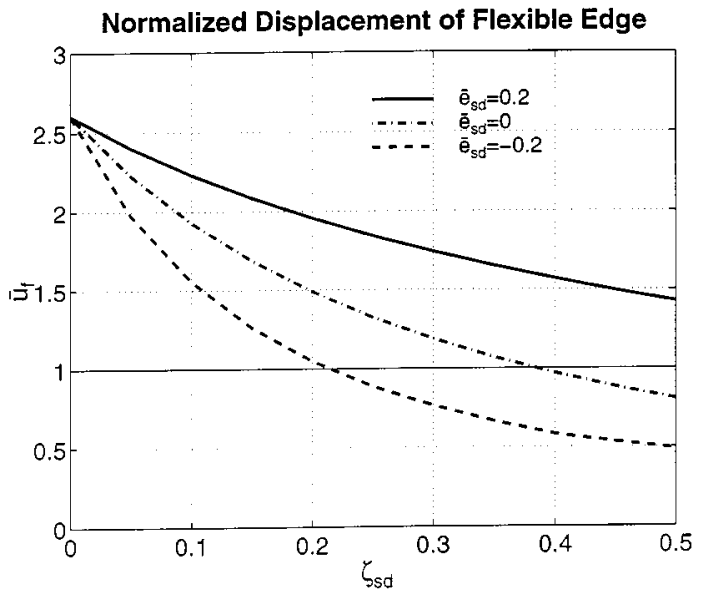

(a)

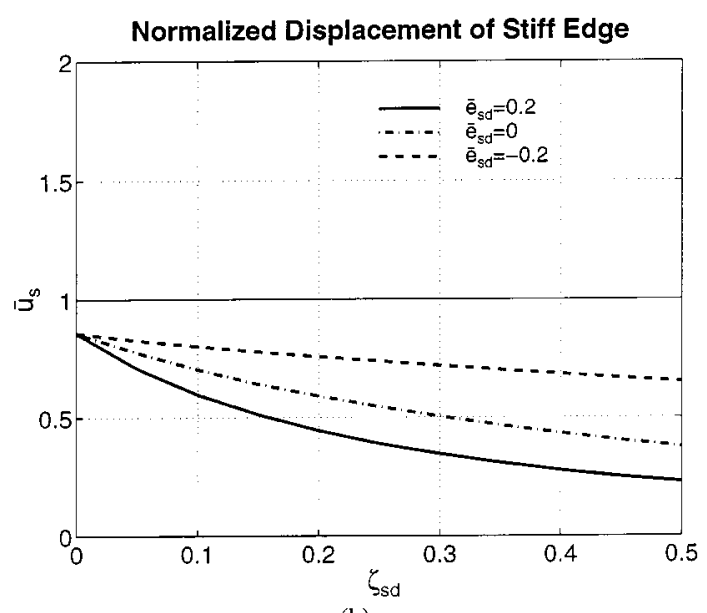

(b)

Figure 11. Normalized edge deformations in asymmetric-plan systems $\left(\bar{e}=0 \cdot 2 ; \Omega_{\theta}=1 ; \alpha=2 ; \zeta=5\right.$ per cent; $\left.T_{y}-=1 \mathrm{~s}\right)$ with supplemental damping $\left(\bar{\rho}_{\text {sd }}=0 \cdot 2\right)$

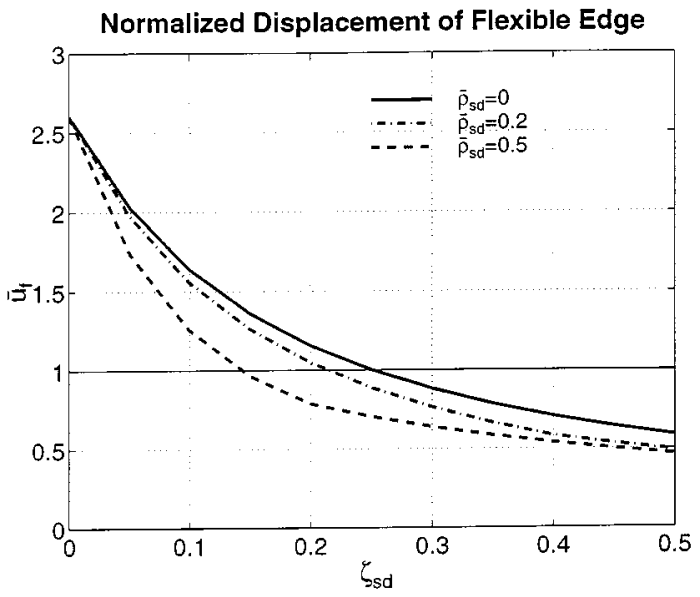

(a)

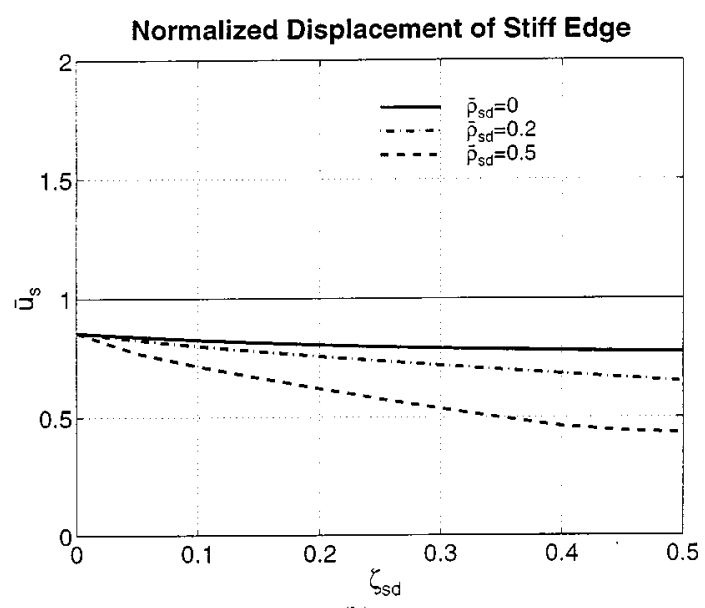

(b)

Figure 12. Normalized edge deformations in asymmetric-plan systems $\left(\bar{e}=0 \cdot 2 ; \Omega_{\theta}=1 ; \alpha=2 ; \zeta=5\right.$ per cent; $\left.T_{y}=1 \mathrm{~s}\right)$ with supplemental damping $\left(\bar{e}_{\mathrm{sd}}=-0 \cdot 2\right)$

\section{Supplemental damping ratio}

Presented in Figures 11 and 12 are the normalized deformations, $\bar{u}_{\mathrm{f}}$ and $\bar{u}_{\mathrm{s}}$, in asymmetric-plan systems against $\zeta_{\text {sd }}$; values of $\zeta_{\text {sd }}$ in the range of $0-0.5$ are considered. The results presented in Figure 11 are for three values of $\bar{e}_{\mathrm{sd}}=0 \cdot 2,0$ and $-0 \cdot 2$ and a fixed value of $\bar{\rho}_{\mathrm{sd}}=0 \cdot 2$, whereas those in Figure 12 are for three values of $\bar{\rho}_{\mathrm{sd}}=0,0.2$ and 0.5 and a fixed value of $\bar{e}_{\mathrm{sd}}=-0.2$. In each of the two figures, the responses were computed for systems with $T_{y}=1 \mathrm{~s}$.

These results show that edge deformations become smaller as supplemental damping $\zeta_{\text {sd }}$ increases, an effect that is stronger for smaller values of $\zeta_{\text {sd }}$. This means that the reduction in edge deformation is greater due to 
the initial 5\% supplemental damping (i.e., increase in $\zeta_{\text {sd }}$ from 0 to $5 \%$ ), compared with the reduction due to an increase in supplemental damping by the same amount at a later stage (i.e., increase in $\zeta_{\text {sd }}$ from 10 to $15 \%$ ). This is also apparent from the reduction in the slope (or flattening) of the curves as $\zeta_{\text {sd }}$ increases. These observations are consistent with previous ones. ${ }^{23}$

The stiff edge deformations are affected to a lesser degree by supplemental damping than the flexible edge deformations, which becomes apparent from the relatively flat curves for $\bar{u}_{\mathrm{s}}$ (Figures 11(b) and 12(b)). In a few cases, for example, $\bar{e}_{\text {sd }}=-0 \cdot 2$ and $\bar{\rho}_{\text {sd }}=0$, the stiff edge deformation is reduced very little as the value of $\zeta_{\text {sd }}$ increases from 0 to 50 per cent (solid curve in Figure 12(b)) compared to a reduction by a factor of nearly five in deformation of the flexible edge over the same range of $\zeta_{\text {sd }}$ (solid curve in Figure 12(a)). The variations of the edge deformations with $\bar{e}_{\text {sd }}$ (Figure 11) and $\bar{\rho}_{\text {sd }}$ (Figure 12) are consistent with the ones noted in the preceding sections.

As mentioned previously, deviations in value of the normalized edge deformation from unity are indicative of the effects of plan asymmetry. This implies that if the effects of plan asymmetry are to be reduced, the asymmetric-plan systems should be modified such that the normalized deformations for both edges are nearly the same and equal to one. In other words, edge deformations in asymmetric-plan systems should be close to deformations of the same edges in the corresponding symmetric-plan systems. The results presented in this paper indicate that the effects of plan asymmetry may be minimized by incorporating FVDs without redistributing the stiffness and/or mass properties. However, a careful selection of the supplemental damper parameters is required for this purpose. For example, for a structure with $T_{y}=1 \mathrm{~s}, \Omega_{\theta}=1, \alpha=2$, and structural eccentricity of 20 per cent of the plan dimension, the flexible edge deformation prior to any modification is more than 2.5 times the deformation of the same edge if the system plan were symmetric, i.e., $\bar{u}_{\mathrm{f}} \approx 2.5$ (Figure 11(a)). This deformation may be reduced such that $\bar{u}_{\mathrm{f}} \approx 1$ by providing $\zeta_{\mathrm{sd}}=20 \%$ through FVDs and distributing them such that $\bar{e}_{\text {sd }}=-0 \cdot 2$ and $\bar{\rho}_{\text {sd }}=0 \cdot 2$. The deformation of the stiff edge, which was about 80 per cent of the deformation of the same edge in the corresponding symmetric plan system, is affected very little by the addition of FVDs for this system.

\section{CONCLUSIONS}

This investigation was focused on seismic behaviour of linearly-elastic, one-storey, asymmetric-plan systems with supplemental viscous damping devices. Identified first were three additional system parameters related to the supplemental damping and its plan-wise distribution: (1) the damping ratio due to supplemental damping devices, $\zeta_{\mathrm{sd}} ;(2)$ the normalized supplemental damping eccentricity, $\bar{e}_{\mathrm{sd}}$; and (3) the normalized supplemental damping radius of gyration, $\bar{\rho}_{\text {sd }}$. Subsequently, the effects of these parameters on the flexible and stiff edges of asymmetric-plan systems were investigated. It was shown that supplemental damping reduced edge deformations. Reductions by a factor of up to about three are feasible, however, the degree of reduction strongly depends on the plan-wise distribution of the supplemental damping. In particular, it was found that:

1. For the same amount of supplemental damping, an asymmetric distribution led to a higher reduction in edge deformations as compared to a symmetric distribution; an additional reduction in the edge deformation by a factor of nearly two, as compared to the symmetric distribution of the supplemental damping, is possible.

2. The large reduction in edge deformations occurred when the CSD was as far away as physically possible from the CM; the CSD should be on the opposite side of the CR (negative $\bar{e}_{\text {sd }}$ ) to obtain a reduction in the flexible edge deformation, whereas it should be on the same side as the CR (positive $\bar{e}_{\text {sd }}$ ) for reduction in the stiff edge deformation.

3. The largest reduction in edge deformations was also obtained when the supplemental damping is distributed as far away from the CSD as possible. 
4. Since $\bar{e}_{\text {sd }}$ and $\bar{\rho}_{\text {sd }}$ cannot physically take on the largest possible values simultaneously, a near optimal reduction may be obtained by using (a) as few dampers as possible in the direction under consideration and locating the outermost dampers at the two edges, and (b) dampers in the perpendicular direction.

5. The effects of the plan-wise distribution of supplemental damping were much more significant for strongly coupled $\left(\Omega_{\theta}=1\right)$ and torsionally-very-flexible $\left(\Omega_{\theta}=0 \cdot 5\right)$ asymmetric-plan systems. Furthermore, these effects were more pronounced for the flexible edge. Although edge deformations of torsionally-very-stiff $\left(\Omega_{\theta}=2\right)$ asymmetric-plan systems were reduced due to supplemental damping, they were essentially unaffected by its plan-wise distribution.

It has also been shown that edge deformations in asymmetric-plan systems can be reduced to levels equal to or smaller than those of the same edges in the corresponding symmetric-plan system by proper selection of the supplemental damping parameters alone, without redistributing the stiffness and/or mass properties of the system.

The findings in this paper are based on the responses of simple, one-storey systems computed for single earthquake excitation. It would be useful to investigate the response behaviour of more complex systems, such as multi-storey buildings, and consider an ensemble of earthquakes in order to gain further confidence in these findings.

\section{ACKNOWLEDGEMENTS}

The author would like to thank Professors Anil Chopra and Andrei Reinhorn for their useful discussion on the subject matter of this paper.

\section{REFERENCES}

1. R. Hejal and A. K. Chopra, 'Earthquake response of torsionally-coupled buildings', Report UCB/EERC-87/20, Earthquake Engineering Research Center, University of California, Berkeley, CA, 1987.

2. R. K. Goel and A. K. Chopra, 'Inelastic seismic response of one-story, asymmetric-plan systems', Report UBC/EERC-90/14, Earthquake Engineering Research Center, University of California, Berkeley, CA, 1990.

3. A. Rutenberg, 'Nonlinear response of asymmetric building structures and seismic codes: A state of the art review', European Earthquake Engng. VI(2), 3-19 (1992).

4. International Association for Earthquake Engineering, Earthquake Resistant Regulations, A World List, 1992 , Tokyo, 1992.

5. I. D. Aiken and J. M. Kelly, 'Earthquake simulator testing and analytical studies of two energy-absorbing systems for multistory structures', Report UBC/EERC-90/03, Earthquake Engineering Research Center, University of California, Berkeley, CA, 1990.

6. K. C. Chang, 'Seismic behavior of steel frame with added viscoelastic dampers', J. Struct. Engng. 121(10), 1418-1426 (1995).

7. K. C. Chang, T. T. Soong, M. L. Lai, E. J. Nielsen, 'Viscoelastic dampers as energy dissipation devices for seismic applications', Earthquake Spectra 9(3), 371-388 (1993).

8. M. C. Constantinou and M. D. Symans, 'Experimental and analytical investigation of seismic response of structures with supplemental fluid viscous dampers', Report No. NCEER-92-0032, National Center for Earthquake Engineering Research, Buffalo, NY, 1992.

9. Y. Fu, 'Frame retrofit by using viscous and viscoelastic dampers', CD-ROM Proc. 11th World Conf. on Earthquake Engineering, Paper No. 428, Elsevier Science Ltd., NY, 1996.

10. N. Gluck, A. M. Reinhorn, J. Gluck and R. Levy, 'Design of supplemental dampers for control of structures', J. Struct. Engng. 122(12), 1394-1399 (1996).

11. R. D. Hanson, 'Supplemental damping for improved seismic performance', Earthquake Spectra 9(3), 319-334 (1993).

12. R. D. Hanson, I. D. Aiken, D. K. Nims, P. J. Richter, and R. E. Bachman, 'State-of-the-art and state-of-the-practice in seismic energy dissipation', Proc. Seminar and Workshop on Base Isolation, Passive Energy Dissipation and Active Control, ATC 17-1, Vol. 2, Applied Technology Council, Palo Alto, CA, 1993, 449-472.

13. K. Kasai and J. A. Munsi, 'Seismic response of viscoelastic frame with yielding members', Proc. 5th National Conference on Earthquake Engineering, Vol. I, Earthquake Engineering Research Institute, Oakland, CA, 1994, 839-848.

14. N. Makris and M. C. Constantinou, 'Spring-viscous damper systems for combined seismic and vibration isolation', Earthquake Engng. Struct. Dyn. 21(8), 649-664 (1992).

15. A. S. Pall, 'Energy dissipation devices for aseismic design of buildings', Proc. Seminar and Workshop on Base Isolation and Passive Energy Dissipation, ATC-17, Applied Technology Council, Palo Alto, California, 1986, 223-232.

16. A. M. Reinhorn, C. Li and M. C. Constantinou, 'Experimental and analytical investigation of seismic retrofit of structures with supplemental damping: Part 1 - Fluid viscous damping devices', Report No. NCEER-95-0001, National Center for Earthquake Engineering Research, Buffalo, NY, 1995. 
17. P. Tsopelas, S. Okamoto, M. C. Constantinou, D. Ozaki and S. Fuji, 'Experimental and analytical study of systems consisting of sliding bearing, rubber restoring force devices, and fluid dampers', Report No. NCEER-94-0002, National Center for Earthquake Engineering Research, Buffalo, NY, 1994.

18. Rasmussen, E. 'Dampers hold sway', Civil Engng. 67(3), 40-43 (1997).

19. A. Arista and R. Gomez, 'Influence of energy dissipation devices on the torsional response of single-story structures', Proc. Seminar and Workshop on Base Isolation and Passive Energy Dissipation, ACT-17-1, Applied Technology Council, Vol. 2, 1993, 651-662.

20. L. Martin and O. A. Pekau, 'Improved performance of friction damped asymmetric structures', Proc. 5th Canadian Conf. on Earthquake Engineering, Montreal, 1995, 927-934.

21. O. A. Pekau and R. Guimond, 'Controlling seismic response of eccentric structures by friction dampers', Earthquake Engng. and Struct. Dyn. 20(6), 505-521 (1991).

22. Z. X. Li and Y. A. He, 'Optimal damper control for 3-dimensional tall buildings under earthquake', Proc. 10th World Conf. on Earthquake Engineering, Vol. 7, 1992, 4159-4164.

23. A. K. Chopra, Dynamics of Structures: Theory and Applications to Earthquake Engineering, Prentice-Hall, Upper Saddle River, NJ, 1995. 